\title{
Knock-down of LRP/LR promotes apoptosis in early and late stage colorectal carcinoma cells via caspase activation
}

\author{
Leila Vania, Thalia M. Rebelo, Eloise Ferreira and Stefan F. T. Weiss ${ }^{*}$ (1)
}

\begin{abstract}
Background: Cancer remains one of the leading causes of death around the world, where incidence and mortality rates are at a constant increase. Tumourigenic cells are characteristically seen to over-express the $37 \mathrm{kDa} / 67 \mathrm{kDa}$ laminin receptor (LRP/LR) compared to their normal cell counterparts. This receptor has numerous roles in tumourigenesis including metastasis, angiogenic enhancement, telomerase activation, cell viability and apoptotic evasion. This study aimed to expose the role of LRP/LR on the cellular viability of early (SW-480) and late (DLD-1) stage colorectal cancer cells.

Methods: siRNA were used to down-regulate the expression of LRP/LR in SW-480 and DLD-1 cells which was assessed using western blotting. Subsequently, cell survival was evaluated using the MTT cell survival assay and confocal microscopy. Thereafter, Annexin V-FITC/PI staining and caspase activity assays were used to investigate the mechanism underlying the cell death observed upon LRP/LR knockdown. The data was analysed using Student's ttest with a confidence interval of 95\%, with $p$-values of less than 0.05 seen as significant.

Results: Here we reveal that siRNA-mediated knock-down of LRP led to notable decreases in cell viability through increased levels of apoptosis, apparent by compromised membrane integrity and significantly high caspase-3 activity. Down-regulated LRP resulted in a significant increase in caspase-8 and -9 activity in both cell lines.

Conclusions: These findings show that the receptor is critically implicated in apoptosis and that LRP/LR downregulation induces apoptosis in early and late stage colorectal cancer cells through both apoptotic pathways. Thus, this study suggests that siRNA-mediated knock-down of LRP could be a possible therapeutic strategy for the treatment of early and late stage colorectal carcinoma.
\end{abstract}

Keywords: Colorectal cancer, Small interfering RNAs, Apoptosis, 37 kDa/67 kDa laminin receptor, LRP/LR, Therapeutics

\section{Background}

Cancer remains one of the main causes of death around the world, where incidence and mortality rates are at a constant increase. According to the World Health Organisation (WHO), over 14 million new cases were diagnosed in 2015, and 8.8 million cancer related deaths were reported [1]. The current study focuses on a particular cancer type known as colorectal cancer. In South Africa, colorectal cancer is found to be the 5th most common cancer [2]. However, globally, it has been

* Correspondence: Stefan.weiss@wits.ac.za

School of Molecular and Cell Biology, University of the Witwatersrand, Private Bag 3, Wits 2050, Johannesburg, Republic of South Africa ranked as the 3rd most common cancer type with over 1.4 million new cases in the year 2015 - contributing to 9.7\% of the total number of cancer cases diagnosed, including 774,000 cancer related deaths [1]. Due to the increasing prevalence and mortality rates of colorectal cancer, it is crucial to develop a novel treatment strategy to combat this disease.

There are several intrinsic and extrinsic factors which contribute to normal cells transforming into cancerous cells. Due to the complexity and diversity of neoplastic diseases, the collective term known as "the hallmarks of cancer" came about in order to provide a better understanding of this disease [3]. These hallmarks show that

(c) The Author(s). 2018 Open Access This article is distributed under the terms of the Creative Commons Attribution 4.0 International License (http://creativecommons.org/licenses/by/4.0/), which permits unrestricted use, distribution, and reproduction in any medium, provided you give appropriate credit to the original author(s) and the source, provide a link to the Creative Commons license, and indicate if changes were made. The Creative Commons Public Domain Dedication waiver (http://creativecommons.org/publicdomain/zero/1.0/) applies to the data made available in this article, unless otherwise stated. 
tumour cells acquire several capabilities that their normal counterparts do not have, including: independent of growth signals, resistance to anti-growth signals, unlimited replicative potential, tissue invasion and metastasis, continuous angiogenesis and apoptosis evasion [3]. In addition, recent studies have shown that cancerous cells also require the help of a particular receptor known as the $37 \mathrm{kDa}$ laminin receptor precursor/ $67 \mathrm{kDa}$ laminin receptor (LRP/LR) to maintain their tumourigenic state [4-9].

LRP/LR, also known as RPSA, is known to assist in numerous physiological processes $[10,11]$. Moreover, the receptor possesses a strong binding affinity for laminin-1, a ligand found in several non-collagenous glycoproteins and is said to play critical roles in cell attachment, cell growth and differentiation [12], cell migration [13] and angiogenesis [14]. Hence, the interaction between LRP/LR and laminin-1 in is seen as an enhancement of tumour growth and progression [15]. In addition, LRP/LR has also been seen to play several other roles such as maintaining ribosomal processing of RNA [16], protein synthesis [17], cell cycle regulation [17] and importantly, cell survival [18].

Several studies have shown that LRP/LR contributes to many other pathological conditions such as microbial infections [19], neurological diseases including Alzheimer's disease [20-22], prion-related diseases [23], as well as numerous other cancer types [10]. Furthermore, Naidoo et al. has also shown that LRP/LR mediates telomerase activity by enhancing hTERT activity, thus, illustrating a novel role for the receptor $[24,25]$. Due to LRP/LR being involved in several of the aforementioned tumourigenic processes, this prompted the investigation of the receptor's role in cellular viability and cell survival. One study has revealed that through silencing LRP/LR via siRNA technology, the viability of cervical (HeLa) [26], liver (Hep3B) [27] and lung (A549) [26] cancer cells was reduced by means of apoptotic induction. Other studies indicated that the viability of breast (MCF-7 and MDA-MB231) [28], oesophageal (WHC01) [28], neuroblastoma (IMR-32) [29], pancreatic (AsPC-1) [29] as well as malignant melanoma cancer cells [30] was also reduced through siRNA-mediated LRP/LR knockdown. Therefore, these studies show the vital role of LRP/LR in apoptosis and maintaining tumour cell survival.

Apoptosis is essential for several other processes within organisms including: tissue homeostasis maintenance, normal development preservation, as well as damaged cell elimination - all involving cells actively committing suicide. Once cells undergo apoptosis, they undergo several morphological and biochemical changes [31]. A biochemical change of importance to apoptosis is the activation of caspases. These caspases may become active through two key pathways and as a result induce apoptosis i.e. the intrinsic mitochondrial pathway and the extrinsic death receptor pathway [31].

Hence, the current study investigated whether siRNA-mediated knock-down of LRP/LR will reduce the viability of early (SW-480) and late (DLD-1) stage colorectal cancer cells. This study revealed that knock-down of LRP/LR using siRNA technology significantly reduces the viability of early and late stage colorectal cancer cells, and proposes that apoptosis is the cause for the notable decreases in cellular viability.

\section{Methods}

A detailed list of suppliers/manufacturers of antibodies, reagents and equipment used to carry out the following experiments is given in the supplementary data section.

\section{Cell culture and conditions}

Authenticated colorectal cancer cell lines SW-480 and DLD-1 were obtained from American Tissue Culture Collection (ATCC) with catalogue numbers ATCC $^{\circledR}$ CCL-228 and ATCC ${ }^{\circledast}$ CCL-221, respectively. Both cancer cell lines were cultured in DMEM/Ham's F-12 (1:1) (GE Lifesciences) together with 10\% Fetal Calf Serum (FCS) (Capricorn Scientific) and $1 \%$ penicillin/streptomycin (Biowest). All cells remained at $37{ }^{\circ} \mathrm{C}$ with $5 \% \mathrm{CO}_{2}$ in a humidified incubator.

\section{siRNA-mediated knock-down of the laminin receptor (LRP/LR)}

Cell counts were performed with the $\mathrm{TC} 20^{\mathrm{Tm}}$ cell counter (Biorad) and cells were seeded at a density depending on the experiment being performed. Cells were allowed to reach $50-70 \%$ confluency prior to transfection. Both cancer cell lines were transfected with ON-TARGETplus SMARTpool Human-RPSA (GE Dharmacon) (targeted towards LRP/LR) - this siRNA will be referred to as siRPSA \#1 and esiRNA-RLUC (serving as the negative control) (Sigma). The appropriate amounts of DharmaFect transfection reagent (GE Dharmacon) and Mission transfection reagent (Sigma) were added to the cells, respectively. The cancer cell lines were likewise transfected with esiRNA-RPSA (Sigma) which is also targeted to LRP/LR (this siRNA will be referred to as siRPSA \#2). Thereafter, cells were incubated for $72 \mathrm{~h}$ at $37{ }^{\circ} \mathrm{C}$. This procedure was performed before any further experiments took place.

\section{SDS-PAGE and western blotting}

To determine siRNA-treated LRP/LR levels in the colorectal cancer cell lines, western blotting was performed. Cell lysates containing $10 \mu \mathrm{g}$ of protein were separated on $12 \%$ sodium dodecyl sulphate polyacrylamide gels via electrophoresis (SDS-PAGE) (Bio-Rad). Thereafter, PVDF membranes (Pall Corporation) were soaked in methanol 
(Associate Chemical Enterprise, ACE) for 2 min followed by a 5 -min incubation in transfer buffer. Proteins were transferred at $300 \mathrm{~V}$ via electro-blotting. The membranes were then blocked for an hour in 0.1\% PBS-Tween in 3\% BSA. Thereafter, the membranes were incubated with the IgG1-iS18 primary antibody which was diluted in blocking buffer (1:5000). The membranes were then washed three times in PBS-Tween (10 min for each wash) followed by an incubation in the appropriate secondary antibody diluted in blocking buffer (1:10000) for $1 \mathrm{~h}$. The membrane was washed three more times with PBS-Tween before adding the chemiluminescent substrate (Biorad) to the membrane in order to detect proteins. In addition, $42 \mathrm{kDa}$ $\beta$-actin served as a loading control. Finally, densitometric analysis was completed in order to quantify protein levels using ImageLab ${ }^{\mathrm{Tm}}$ software.

\section{MTT assay}

The MTT assay is a valid assay for determining cell viability employed in various cancer studies [28-30, 32-35]. Before transfections took place, $1 \times 10^{4}$ cells $/ \mathrm{ml} \mathrm{SW-480}$ and DLD-1 cells were seeded on 24-well plates. After transfection, cells were incubated at $37{ }^{\circ} \mathrm{C}$ for $72 \mathrm{~h}$, followed by the addition of $1 \mathrm{mg} / \mathrm{ml}$ of MTT [100 $\mu \mathrm{g}$ of MTT (Duchfei Biochemic) being dissolved in $1 \mathrm{X}$ PBS (Gibco)] to all wells. This was followed by a further incubation at $37{ }^{\circ} \mathrm{C}$ for $2 \mathrm{~h}$. Thereafter, the media from each well containing MTT was removed and $500 \mu \mathrm{l}$ DMSO was added to dissolve the residual formazan crystals (Merck Millipore). The resultant absorbance was measured at $570 \mathrm{~nm}$. This procedure was performed for controls as well which included: untreated cells, PCA (Protocatechuic acid) positive control (Aldrich Chemistry) treated cells as well as esiRNA-RLUC negative control treated cells.

\section{Assessment of nuclear morphological changes - Confocal microscopy with Airyscan}

In order to evaluate nuclear morphology post knock-down of LRP/LR via siRNA technology, confocal microscopy was used. Early and late stage colorectal cancer cells were seeded onto coverslips at a cell density of $1 \times 10^{5}$ cells $/ \mathrm{ml}$. After transfection, the cells were fixed in 4\% PFA (Associated Chemical Enterprise, ACE) for 15 min prior to 3 washes with PBS. Once remaining PBS was blotted off after washing, cells were then incubated with $0.1 \%$ Triton-X for 20 min for permeabilization of the cell membrane. The cells were then washed twice followed by the addition of DAPI nuclear stain (Sigma) diluted in PBS (1:100) onto each coverslip and incubated for $8 \mathrm{~min}$ in the dark. Once stained, the coverslips were washed twice in PBS prior to each coverslip being mounted on a microscope slide with fluoromount (Sigma). The microscope slides were left to set for $45 \mathrm{~min}$ in the dark, after which they were maintained at $4{ }^{\circ} \mathrm{C}$. Note: untreated cells were used as a control, esiRNA-RLUC as a negative control and PCA as a positive control. Airyscan is a technique used to enhance confocal laser scanning microscopy. It has been shown that total resolution is improved by a factor of 1.7 in all spatial directions i.e. a resolution of $140 \mathrm{~nm}$ laterally and $400 \mathrm{~nm}$ axially can be achieved. The Airyscan was used to further analyse the nuclear morphological changes observed after LRP/LR down-regulation (Zeiss LSM 780).

\section{Annexin V-FITC/7AAD assays}

This experiment was performed as per the manufacturer's directions (Beckman Coulter). Both SW-480 and DLD-1 cells were seeded at a cell density of $2 \times 10^{6}$ cells/ml before transfection. After $72 \mathrm{~h}$ incubation at $37{ }^{\circ} \mathrm{C}$, cells were subjected to trypsinization with trypsin/EDTA (Biowest) followed by washes with cold PBS. Thereafter, cells were centrifuged at $5000 \mathrm{rpm}$ for $5 \mathrm{~min}$ was performed, after which pellets were resuspended in $1 \mathrm{X}$ annexin-binding buffer (BD Sciences). Thereafter, $10 \mu \mathrm{l}$ of Annexin V-FITC (BD Sciences) solution and $5 \mu \mathrm{l}$ of PI viability dye were added to each cell suspension which was followed by a 15 -min incubation on ice in the dark. Subsequently, $400 \mu \mathrm{l}$ of ice-cold $1 \mathrm{X}$ annexin binding buffer was added to the samples for $30 \mathrm{~min}$ and all resulting cell suspensions were reviewed using the BD Accuri C6 flow cytometer. Note: esiRNA-RLUC was the negative control and PCA was the positive control.

\section{Caspase-3, -8 and -9 activation assays}

Caspase-3,-8 and -9 assays were completed as per the manufacturer's directions (Merck Millipore). Cells were seeded at a cell density of $1 \times 10^{6}$ cells $/ \mathrm{ml}$ before transfection. Cells were then centrifuged at $1200 \mathrm{rpm}$ for $10 \mathrm{~min}$, followed by pellet resuspension in $50 \mu \mathrm{l}$ of lysis buffer. The samples were then incubated for $10 \mathrm{~min}$ on ice, followed by a further centrifugation at $10000 \mathrm{x} g$ for 5 min. While the pellet was discarded, the supernatant was placed into a new microcentrifuge tube and put on ice. Thereafter, a $\mathrm{BCA}^{\mathrm{Tm}}$ assay was performed in order to obtain the supernatant's protein concentration. This was followed by $200 \mu \mathrm{g}$ of protein being diluted per sample, prior to being added to wells of a 96-well plate. Once this was completed, $20 \mu \mathrm{l}$ of $5 \mathrm{X}$ assay buffer was added to every sample. Thereafter, $10 \mu \mathrm{l}$ of peptide substrate was added followed by incubation at $37{ }^{\circ} \mathrm{C}$ for $2 \mathrm{~h}$. Finally, the absorbance was read at $405 \mathrm{~nm}$. Note: esiRNA-RLUC treated cells served as a negative control and PCA treated cells were used as a positive control.

\section{Statistical evaluation}

In order for accurate data analysis, Student's t-test had to be utilized, with a confidence interval of $95 \%$. 
Furthermore, $p$-values greater than 0.05 were seen as non-significant. To measure the degree of association between LRP/LR levels and apoptotic induction as well as cellular viability, Pearson's correlation coefficient was calculated. A positive coefficient shows a directly proportional relationship between the two variables (where values close to 1 indicates a highly positive correlation)

\section{Results}

siRNA technology successfully results in knock-down of LRP expression and reductions in cellular viability in early and late stage colorectal cancer cells

To understand the effect LRP/LR expression has on early and late stage colorectal cancer cell viability, down-regulation of the receptor had to be performed. Once early (SW-480) and late (DLD-1) cells were transfected with siRPSA \#1 (targeted towards the $37 \mathrm{kDa}$ LRP mRNA), evaluation of Western blotting and densitometry was performed. Densitometry showed that LRP was significantly knocked down in both SW-480 and DLD-1 cells when transfected with siRPSA \#1. The SW-480 and DLD-1 transfected cells exhibited a 75 and $78 \%$ decrease in LRP expression, respectively, when compared to cells that were not transfected, since these LRP levels were set to $100 \%$ (Fig. 1a and b). Additionally, to determine whether the reduction in LRP expression had resulted due to siRPSA \#1-mediated LRP knock-down and not just an off-target effect, an alternative siRNA that targets another region of LRP was utilised. When comparing them to the non-transfected cells, both early (SW-480) and late (DLD-1) stage colorectal cancer cells displayed a knock-down of 72 and $61 \%$ in LRP expression, respectively (Fig. 1c and d). SW-480 and DLD-1 cells transfected with the esiRNA-RLUC showed no significant LRP knock-down when comparing them to non-transfected cells. Once LRP expression was successfully down-regulated using siRNA technology, its effect on the viability of both cell lines were observed. MTT assays were performed which showed that when SW-480 and DLD-1 cells were transfected with siRPSA \#1, cell viability was significantly decreased in contrast to cells that were not transfected, indicating that siRNA-mediated knock-down of the receptor leads to reductions in cell viability in both early and late stage colorectal cancer cell lines. The MTT assays revealed that SW-480 and DLD-1 cells treated with siRPSA \#1 displayed a 60 and 55\% decrease, respectively, in comparison to the cells that were not transfected (Fig. 1g). Additional MTT assays were performed to evaluate whether siRPSA \#2-mediated LRP knock-down also influenced the viability of SW-480 and DLD-1 cells. Post treatment of cells with siRPSA \#2 was found to reduce the viability significantly by 44 and $89 \%$ in both SW-480 and DLD-1 cells, respectively, when comparing them to the cells which were not transfected (Fig. 1g). Additionally, treating SW-480 and DLD-1 cells with the negative control siRNA, esiRNA-RLUC, indicated no notable change in cell viability in comparison to cells that were not transfected (Fig. 1g).

\section{Knock-down of LRP expression via siRNA technology leads to changes in nuclear morphology signifying apoptosis}

To determine whether the decreases in cell viability after treatment with siRPSA \#1 was caused by apoptotic induction, nuclear morphological changes were studied by confocal microscopy and Airyscan. siRPSA \#1 treated early (SW-480) stage colorectal cancer cells exhibited nuclear morphological changes in the form of condensed nuclei and reduced nuclear size (Fig. 2d), when compared to the nuclei of cells that were not transfected (Fig. 2a). Late (DLD-1) stage colorectal cancer cells transfected with siRPSA \#1 presented nuclear morphological changes such as cellular fragmentation into membrane-bound bodies, weakened membrane integrity and membrane blebbing and (Fig. 2h), in contrast to nuclei of cells that were not transfected (Fig. 2e). Membrane blebbing was confirmed for the DLD-1 cells by bright field microscopy (Additional file 1: Figure S1). Results obtained for siRPSA \#1 treated cells were consistent with those of the positive control, PCA (Fig. 2c and g). In addition, upon treatment with esiRNA-RLUC, both cell lines did not reveal any morphological changes in nuclei, when comparing them to cells that were not transfected (Fig. 2b and f).

\section{siRNA-mediated knockdown of LRP expression induces apoptosis in early and late stage colorectal cancer cells} Due to confocal microscopy proposing that the knock-down of LRP expression in early (SW-480) and late (DLD-1) stage colorectal cancer cells leads to morphological changes of the nuclei (a key feature of cells undergoing apoptosis), Annexin-V/PI assays had to be performed to confirm this result quantitively.

SW -480 cells treated with siRPSA \#1 revealed $36.6 \%$ of cells undergoing early apoptosis, while $44.1 \%$ of cells underwent late apoptosis (Fig. 3.1d), in contrast to cells that were not transfected (Fig. 3.1a). Transfected DLD-1 cells with siRPSA \#1 resulted in $10.0 \%$ of cells undergoing early apoptosis while $74.3 \%$ of cells underwent late apoptosis (Fig. 3.1h) when compared to cells that were not transfected (Fig. 3.1e) Additionally, upon treatment with esiRNA-RLUC, SW-480 and DLD-1 cell lines did not undergo apoptosis (Fig. 3.1b and f). PCA positive control showed most cells underwent late apoptosis (Fig. 3.1c and g. Further statistical analysis confirmed 
a

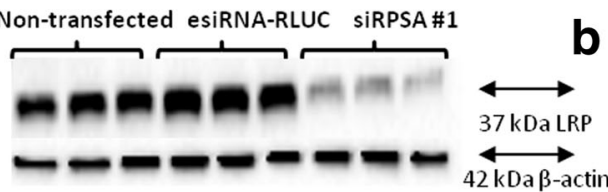

C
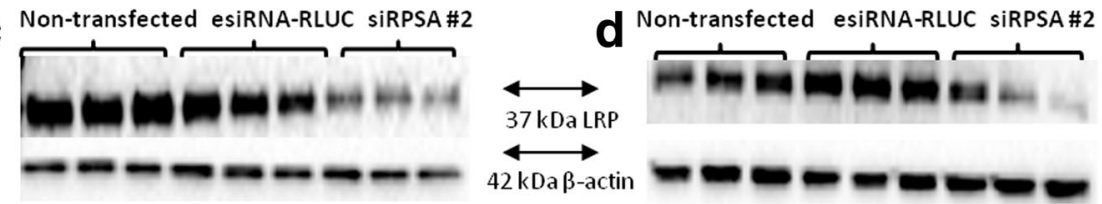

e

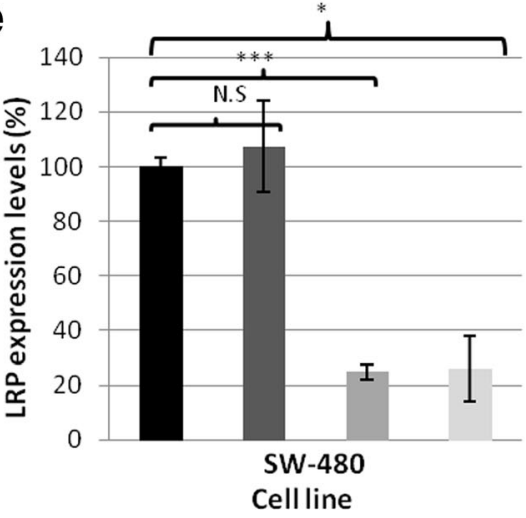

f

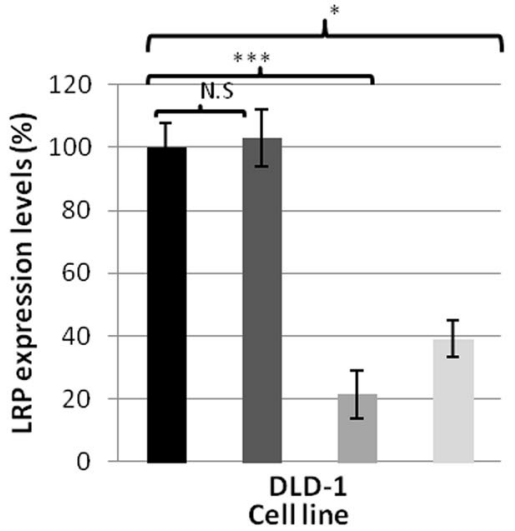

g

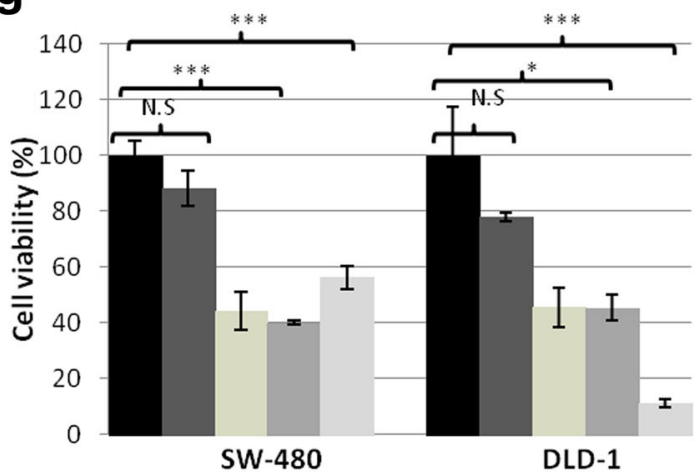

DLD-1

Cell lines

Fig. 1 The effect of siRNA-mediated knock-down on LRP expression and the viability of early (SW-480) and late (DLD-1) stage colorectal cancer cells. a), c) and e) Upon transfection of SW-480 with siRPSA \#1 and siRPSA \#2, significant 75 and $72 \%$ decreases in LRP expression levels was revealed, respectively, in contrast to cells that were not transfected. Densitometric analysis of LRP levels was performed where levels of the non-transfected cells were set to $100 \%$. ${ }^{* *} p=0.0001,{ }^{*} p=0.02$ N.S.: $p>0.05 . \mathbf{b}$ ), d) and f) siRPSA \#1 and siRPSA \#2 transfection in DLD-1 cells caused significant 79 and $61 \%$ decreases in LRP expression levels, respectively, when comparing them to cells that were not transfected. Densitometry displayed no significant difference in LRP expression between non-transfected and negative control esiRNA-RLUC transfected cells for both cell lines. ${ }^{* *} p=0.0005,{ }^{*} p=0.02$ N.S.: $p>0.05$. g) MTT assays were performed to assess SW-480 and DLD-1 cell viability, upon treatment with siRPSA \#1 and siRPSA \#2. Non-transfected cell value were set to 100\%. It was found that upon siRPSA \#1 transfection, SW-480 and DLD-1 cells exhibited a significant decrease of 60 and $55 \%$ in cellular viability, respectively, in contrast to cells that were not transfected. It was also revealed that when the SW-480 and DLD-1 were transfected with siRPSA \#2, there were significant reductions of 44 and $89 \%$ in cellular viability, respectively, when compared to non-transfected cells. Both cell lines showed no noteworthy differences in cell viability when treated with the negative control esiRNA-RLUC. PCA was used as the positive control. SW-480: siRPSA \#1:*** $p=0.0008$, siRPSA \#2:*** $p=0.0004$ DLD-1: siRPSA $\# 1: * p=0.01$, siRPSA \#2:*** $p=0.0009$, N.S. : $p>0.05$, non-significant. All graphs represent an average of three biological and three technical repeats 


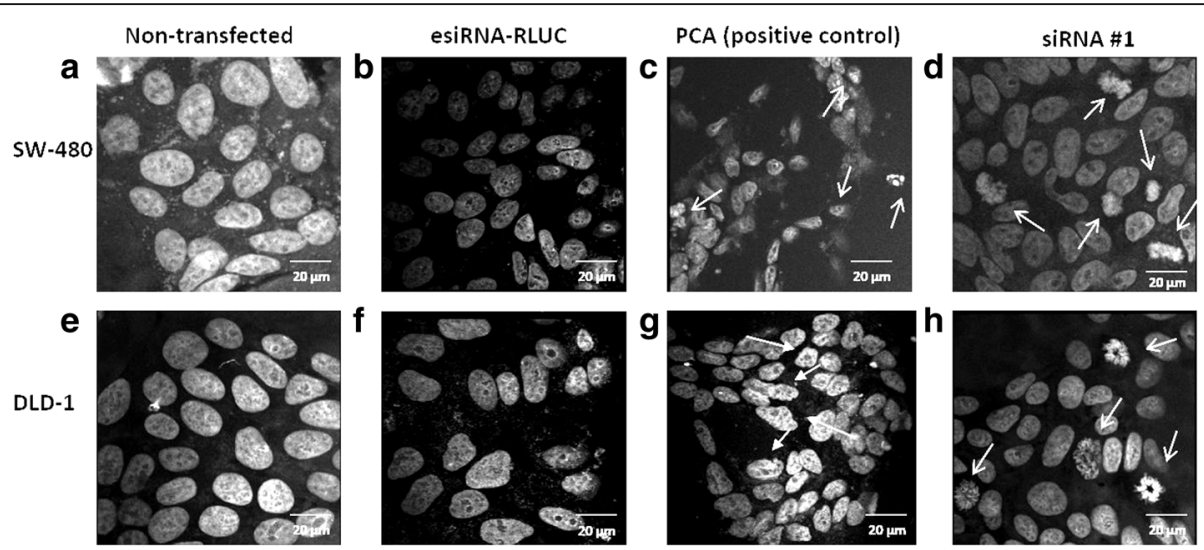

Fig. 2 The effect of siRNA-mediated knock-down of LRP on nuclear morphology of early (SW-480) and late (DLD-1) stage colorectal cancer cells. Confocal microscopy with Airyscan analysis was completed to investigate changes in nuclear morphology upon siRNA treatment. a and e) Non-transfected SW-480 and DLD-1 cells displayed large nuclei with healthy membrane integrity. $\mathbf{b}$ and $\mathbf{f}$ ) SW-480 and DLD-1 cells transfected with negative control esiRNA-RLUC showed parallel characteristics to the non-transfected cells with no changes in nuclear morphology. c) SW-480 cells treated with positive control PCA revealed nuclei that underwent apoptotic body formation. d) SW-480 cells transfected with siRPSA \#1 exhibited nuclear shrinkage and condensed nuclei, proposing induction of apoptosis. g) DLD-1 cells treated with PCA displayed weakened membrane integrity and condensed nuclei. $\mathbf{h}$ ) DLD-1 cells transfected with siRPSA \#1 showed formation of apoptotic bodies and weakened membrane integrity. Images were obtained at a 630X magnification and Airy scan analysis was applied to each image. Scale bars are indicative of $20 \mu \mathrm{m}$

a significant increase in apoptotic cells when treated with siRPSA \#1, in contrast to non-transfected cells in both cell lines (Fig. 3.2).

\section{siRNA-mediated knock-down of LRP expression causes a notable increase in caspase- 3 activity}

For additional validation of apoptosis occurring in early (SW-480) and late (DLD-1) stage colorectal cancer cells once LRP has been down-regulated, caspase-3 activity assays were completed. Post transfection with siRPSA \#1, SW-480 cells were found to have a significant 4-fold increase in caspase- 3 activity, in comparison to cells that were not transfected (Fig. 4a). DLD-1 cells treated with siRPSA \#1 displayed a 5-fold increase in caspase-3 activity in contrast to non-tranfected cells (Fig. 4a). Furthermore, no differences in caspase-3 activity was seen when both cell lines were treated with esiRNA-RLUC (Fig. 4a). PCA positive control displayed a 3-fold and 5-fold increase in caspase- 3 activity was observed in SW-480 and DLD-1 cells, respectively (Fig. 4a).

siRNA-mediated knock-down of LRP results in significant increases in caspase- 8 and caspase- 9 activity in early and late stage colorectal cancer cells

Caspase-3 activation occurs through both apoptosis pathways (intrinsic and extrinsic) hence, further insight of how the receptor aids in tumourigenic cell survival was required; therefore caspase- 8 and -9 activity assays were performed. These assays determine whether treatment with siRPSA \#1 leads to the activation of the extrinsic pathway (facilitated through caspase-8) or the intrinsic pathway (facilitated through caspase-9) in each of the cell lines. SW-480 and DLD-1 cells transfected with siRPSA \#1 were found to undergo a 4-fold increase in caspase- 8 activity, in comparison to cells that were not transfected (Fig. 4b). Moreover, SW-480 cells and DLD-1 cells indicated a significant 7-fold and 4-fold increase in caspase- 9 activity, respectively, in comparison to cells that were not transfected (Fig. 4c). Moreover, both cell lines transfected with esiRNA-RLUC displayed no differences in caspase- 8 and -9 activity in comparison to cells that were not transfected (Fig. 4b).

\section{Discussion}

LRP/LR has gained a large amount of interest due to the many roles it plays in the cell. Particularly, the receptor's over-expression in several cancer cell types as well as its contribution in tumourigenesis has become a target area for research. LRP/LR has been seen to assist with several tumourigenic processes including tumour adhesion and invasion (metastasis), angiogenic enhancement as well as apoptotic evasion [3]. Additionally, since LRP/LR is not limited to the cell surface but also localized in the perinuclear region, cytosol and nucleus, it is able to perform many intracellular and extracellular physiological roles including maintaining cell viability, cell adhesion, cell growth and migration, cell cycle regulation, ribosomal anchorage to microtubules, pre-rRNA processing and protein synthesis. Thus, cancerous cells are found to over-express LRP/LR, thereby exploiting these functions, resulting in the development of the abovementioned tumourigenic processes. Moreover, a recent study 


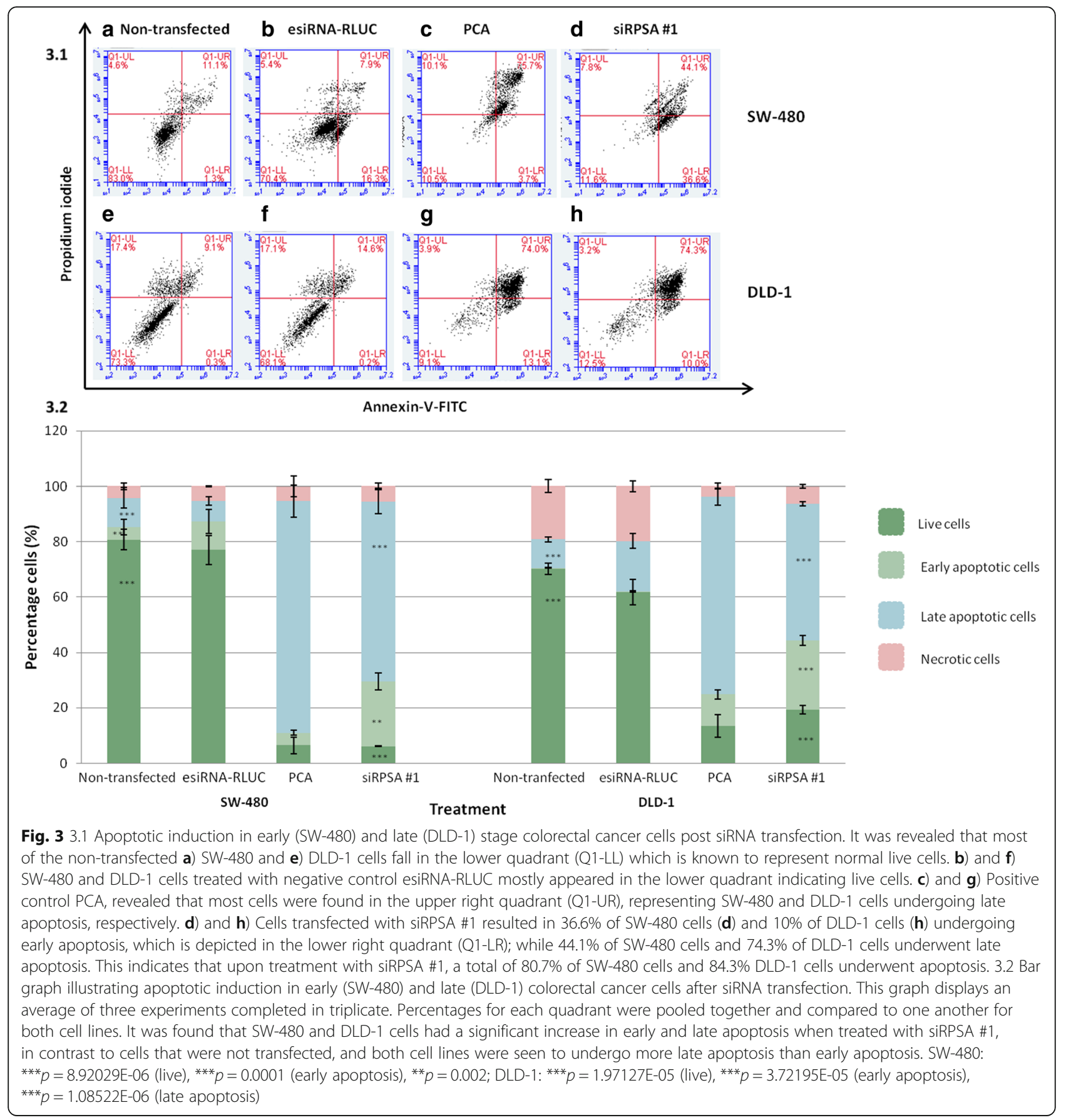

performed by Vania et al. showed that there were significantly higher levels of the receptor in late (DLD-1) stage colorectal cancer cells, compared to the early (SW-480) stage - indicating that LRP expression also increases in the course of malignant transformation [4].

To gain insight into how LRP/LR maintains cell viability, siRNA technology was used to knock-down LRP expression (Fig. 1) and evaluate this effect on cell viability of SW-480 and DLD-1 cells. Due to siRPSA \#1 only targeting the mRNA of the $37 \mathrm{kDa}$ laminin receptor precursor (LRP) form, it was employed to down-regulate LRP in this study (see Additional file 1: Table S1). Cells treated with siRPSA \#1 resulted in significant decreases in LRP down-regulation. Furthermore, a high correlation of 0.91 for SW-480 and 0.96 for DLD-1 was observed between total levels of LRP before and after siRPSA \#1 transfection (Table 1). This high and positive correlation suggests that the level of LRP expression is indeed influenced by siRNA 

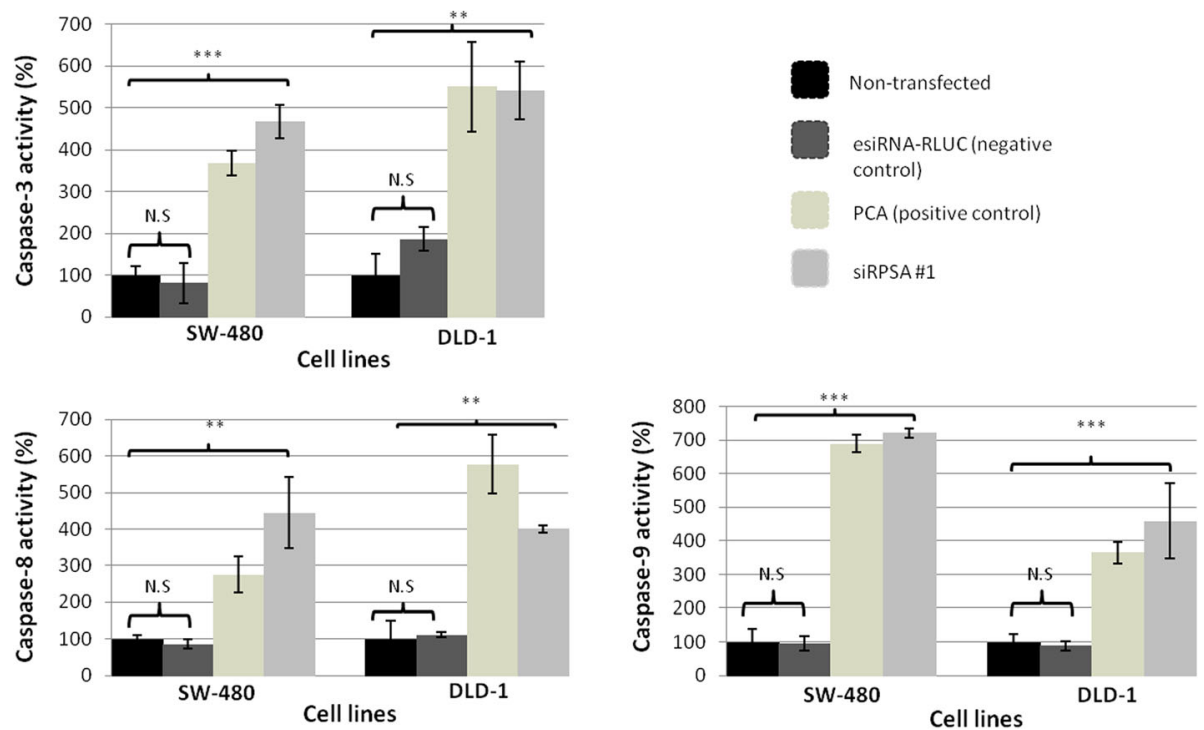

Fig. 4 The effect of siRNA-mediated LRP knock-down on caspase-3, - 8 and -9 activity in early (SW-480) and late (DLD-1) stage colorectal cancer cells. a) Upon treatment of SW-480 and DLD-1 cells with siRPSA \#1, a significant 4-fold and 5-fold increase in caspase-3 activity was revealed, respectively, in comparison to cells that were not transfected (set to 100\%). Both cell lines showed no significant difference in caspase-3 activity between cells transfected with negative control esiRNA-RLUC and non-transfected cells. PCA was used as a positive control. SW-480:*** $p=0.0007$ and DLD-1: ${ }^{* *} p=0.0059$. N.S: $p>0.05$, non-significant. b) siRPSA \#1 transfected SW-480 and DLD-1 cells both displayed a 4-fold significant increase in caspase-8 activity, compared to cells that were not transfected (set to 100\%). Both cell lines showed no significant difference in caspase-8 activity between cells transfected with the esiRNA-RLUC and non-transfected cells. SW-480: ${ }^{* *} p=0.0083$ and DLD-1: ${ }^{* *} p=0.002$. N.S: $p>0.05$, non-significant. C) SW-480 and DLD-1 cells transfected with siRPSA \#1 showed a significant 7-fold increase and 4-fold increase, respectively, in contrast to cells that were not transfected. Both cell lines showed no significant difference in caspase- 9 activity between cells transfected with the esiRNA-RLUC and non-transfected cells. SW-480:*** $p=0.0001$ and DLD-1: ${ }^{* * *} p=0.0008$. N.S.: $p>0.05$, non-significant. This data represents three biological replicates which were completed in triplicate

treatment i.e. lower levels of LRP expression prior to treatment with siRNA leads to more LRP knockdown post treatment with siRNA.

To validate that the observed knock-down was not due to off target effects, SW-480 and DLD-1 cells were both treated with an alternative siRNA, siRPSA \#2. This siRNA targets a specific region of $37 \mathrm{kDa}$ LRP mRNA i.e. nucleotides 521-929 (Table 1). Upon treatment with siRPSA \#2, both cell lines showed significant decreases in LRP knock-down in contrast to cells that were not transfected (Fig. 1). These results validated that LRP was being down-regulated and was not just an off-target effect. In addition, the correlation between total LRP levels before and after siRPSA \#2 treatment was found to be high (sees Additional file 1: Table S2).
To further investigate the receptor's role in maintaining cell viability, an MTT assay was employed to investigate the effect of treatment with siRPSA \#1 and siRPSA \#2 on cell viability. A significant decrease in viability was observed for both SW-480 and DLD-1 cells after LRP down-regulation (Fig. 1). These reductions in cellular viability correlate with the decreased levels of LRP observed after siRNA-mediated down-regulation, signifying the receptor's vital role in the survival of SW-480 and DLD-1 cells (Table 1). It has been discovered that LRP/ LR localised in the nucleus allows for chromosome stability maintenance via interactions with the Midkine heparin-binding growth factor; well-known for enhancing cell proliferation, migration and survival [36]. In addition, several cancer types are showed to have an

Table 1 Assessment of correlation between levels of siRNA-mediated LRP knockdown and viability, apoptotic levels and caspase-3 activity of early (SW-480) and late (DLD-1) stage colorectal cancer cells, using Pearson's correlation co-efficients (R)

\begin{tabular}{lll}
\hline Cell line & SW-480 & DLD-1 \\
\hline Correlation between total LRP levels before and after siRPSA \#1 transfection (R-value) & 0.91 & 0.96 \\
Correlation between siRPSA \#1-mediated LRP knockdown and reduction in cell viability (R-value) & 0.99 & 0.98 \\
Correlation between siRPSA \#2-mediated LRP knockdown and reduction in cell viability (R-value) & 0.99 & 0.93 \\
Correlation between total levels of apoptosis and total LRP levels after siRPSA \#1 transfection (R-value) & 0.99 \\
Correlation between increases in caspase-3 activity and total LRP levels after siRPSA \#1 (R-value) & 0.94 \\
\hline
\end{tabular}


up-regulated expression of Midkine, which results in the promotion of cell survival factors as well as obstruction of apoptosis through caspase-3 inhibition [37]. However, by targeting LRP expression through siRNA technology, LRP/LR-Midkine interactions may decrease, and as a result decrease cell viability.

To establish whether the observed decrease in SW-480 and DLD-1 cell viability post LRP knockdown was due to cell death caused by apoptosis, confocal microscopy was used to evaluate nuclear morphology. Both cell lines revealed several changes in nuclear morphology which were all characteristic of apoptosis including: nuclear condensation, reduced nuclear size and the formation of membrane-bound bodies - when treated with siRPSA \#1 (Fig. 2). It is known that nuclear structures are maintained via the binding of histones to perinuclear and nuclear LRP/LR, thus when the receptor is down-regulated, loss of membrane integrity and distorted nuclear morphology is evident [38].

Although confocal microscopy provided a visual indication that apoptosis was occurring, additional quantification and affirmation of apoptotic induction was needed. This was made possible by means of Annexin V-FITC/PI assays. Non-transfected and negative control esiRNA-RLUC-transfected SW-480 and DLD-1 cells showed negative staining for Annexin-V - indicating live cells. On the other hand, siRPSA \#1-treated and PCA-treated SW-480 and DLD-1 cells exhibited positive staining for Annexin-V or Annexin-V and PI, indicating early and late stage apoptosis, respectively. This shift of Annexin-V staining from negative to positive shows that siRPSA \#1-mediated down-regulation in SW-480 and DLD-1 cells activates membrane asymmetry loss and a membrane-flip reaction involving the externalization of phosphatidylserine (PS) on the outer leaflet of the plasma membrane - allowing these cells to be taken up by phagocytes [39]. Thus, these findings together with the nuclear morphological changes observed, confirm that siRNA-mediated down-regulation of LRP/LR leads to the induction of apoptosis in SW-480 and DLD-1 cells. In addition, the correlation (Table 1) between total levels of LRP after siRPSA \#1 treatment and total levels of apoptosis for both cell lines was found to be high (Figs. 1 and 3.1), further reiterating LRP expression affects cell viability and apoptosis.

Sustantad et al. found that when LRP/LR is down-regulated via siRNA technology, not only did the cell viability of cervical cancer (HeLa) cells and lung (A549) cancer cells decrease but caspase-3 activity was increased in both cell lines [27]. Caspase-3, an effector caspase, is responsible in executing the hallmarks of apoptosis which includes the afore-mentioned nuclear morphological changes by cleaving substrates [40]. Therefore, upon apoptotic induction, caspase- 3 activity is found to be significantly increased. Hence, to further establish that apoptosis was indeed occurring and whether caspases were activated after treatment with siRPSA \#1, caspase-3 assays were performed. Down-regulation of LRP caused a distinct increase in caspase- 3 activity in SW-480 and DLD-1 cells when compared to the cells that were not transfected. Furthermore, the correlation between the total levels of LRP after siRPSA \#1-mediated knockdown (Fig. 1) and increases in caspase-3 activity (Fig. 4) was high in both cell lines (Table 1). These results noticeably point to the induction of apoptosis in SW-480 and DLD-1 cells due to the silencing of LRP though siRNA technology.

It has previously been shown that interactions between LRP/LR and focal adhesion kinase (FAK) are made possible via the binding of the receptor to laminin-1. Furthermore, LRP/LR-FAK interactions were seen to be involved in activating cell signalling cascades such as MEK/ERK $1 / 2$ and PI3-kinase/AKT as well as up-regulating the anti-apoptotic protein, Bcl-2 [41]. This ultimately leads to the inhibition of apoptosis of cancerous cells. Hence, we suggest that silencing LRP/LR through the use of siRNA technology as performed in the current study, impedes the LRP/LR-FAK interaction, and in this way apoptosis is induced. Furthermore, LRP/ LR has been shown to have a direct relationship with the MAPK signalling pathway - where decreased levels of LRP causes a response in the pathway - resulting in cell stress and ultimately, cell death [10]. Another reason which could have led to apoptotic induction through siRNA-mediated knock-down of LRP is the receptor's role in ribosomal processing. Research has shown that LRP/LR is involved in processing $21 \mathrm{~S}$ pre-rRNA into mature 18S rRNA, also known as biogenesis of ribosomes [16]. LRP/LR has also been shown to associate with pre-40S ribosomal subunits, providing nucleolar exits for the subunits, thereby facilitating protein synthesis [42]. We propose that the LRP down-regulation performed in this study may have hampered formation of the ribosome as well as the resultant translation of proteins required for correct cellular functioning, ultimately leading to apoptosis. Furthermore, LRP has also been seen to play a key role in the cell cycle thus, down-regulating the receptor could have resulted in the induction of G1-phase arrest in the colorectal cancer cells, aiding in apoptosis [10].

Since both the MEK/ERK $1 / 2$ and PI3-kinase/AKT cell signalling cascades are known to inhibit both apoptotic pathways, caspase- 8 and caspase- 9 assays were performed to determine if these caspases are activated upon siRPSA \#1-mediated LRP/LR knock-down. Both early and late stage colorectal cancer cell lines were found to have higher caspase- 8 activity post transfection with siRPSA \#1, in contrast to cells that were not transfected. 
Caspase-8 plays a vital role in the extrinsic apoptotic signalling pathway through death receptors, thus it is suggested that siRNA-mediated LRP knock-down induces the apoptotic process in SW-480 and DLD-1 cells extrinsically. Moreover, this study revealed that SW-480 cells and DLD-1 also have increased in caspase-9 activity after LRP down-regulation, in contrast to cells that were not transfected. Caspase- 9 plays a critical role in the intrinsic apoptotic signalling pathway, proposing that siRNA-mediated LRP knock-down also initiates apoptosis in SW-480 and DLD-1 cells through the intrinsic pathway.

The intrinsic and extrinsic pathways interconnect with each other at several levels and can both be influenced by similar factors. In fact, one study showed that activated extrinsic caspase- 8 stimulated the release of cytochrome $c$ and apoptosome formation and ultimately activation of the intrinsic pathway [43, 44]. A potential reason as to why SW-480 and DLD-1 cells experience apoptosis through both apoptotic pathways may be that these colorectal cancer cells undergo a mechanism known as retaliatory caspase activation where the two apoptotic pathways are found to use a feedback amplification loop in order to activate one another [45]. Specifically, activated caspase- 9 initiates and proteolytically cleaves caspase- 3 , also leading to caspase- 8 activation $[45,46]$. Moreover, due to SW-480 and DLD-1 cells undergoing both apoptotic pathways, it can be said that down-regulated LRP/LR possibly hampers both anti-apoptotic signalling pathways on account of the reduced interaction of phosphorylated FAK and LRP/LR.

\section{Conclusions}

This study shows that down-regulating LRP via siRNA technology significantly decreases the viability of early (SW-480) and late (DLD-1) stage colorectal cancer cells through the induction of apoptosis. Moreover, SW-480 and DLD-1 cells underwent apoptosis through both apoptotic pathways. It is possible that cell signalling cascades are involved in inducing apoptosis, however, the exact mechanism is unclear. These findings demonstrates the critical function LRP/LR plays in maintaining the viability of both early and late stage colorectal cancer cells. In addition, these findings emphasize the therapeutic potential of siRNAs targeted against LRP, which could be used as a possible tool in treating early and late stage colorectal cancer.

\section{Additional file}

Additional file 1: Figure S1. Late stage (DLD-1) colorectal cancer cells show membrane blebbing and reduced nuclei post transfection with siRPSA \#1 using bright field microscopy. A) and B) Non-transfected and esiRNA-RLUC (negative control) transfected cells are found to be large with uncompromised membrane integrity. C) and B) siRPSA \#1-transfected and PCA (positive control) treated cells are found to have a reduced size together with compromised membrane integrity i.e. membrane blebbing and condensed nuclei - all indicative of apoptosis occurring. Images were obtained at 200X magnification. Scale bars are indicative of $20 \mu \mathrm{m}$.

Table S1. Sequence of Human-RPSA, esiRNA-RPSA and control siRNA-RLUC used for down-regulation of LRP/LR. Table S2. Pearson's correlation co-efficients (R) between total LRP levels prior to and post transfection with esiRNA-RPSA (DOCX $425 \mathrm{~kb})$

\section{Abbreviations}

ATCC: American type culture collection; BCA: Bicinchoninic acid; BSA: Bovine serum albumin; $\mathrm{CO}_{2}$ : Carbon dioxide; DMEM: Dulbecco's Modified Eagle's medium; DMSO: Dimethyl sulfoxide; ERK: Extracellular signal-regulated kinases; FAK: Focal adhesion kinase; FCS: Fetal calf serum; FITC: Fluorescein isothiocyanate; HRP: Horseradish peroxidase; IgG: Immunoglobulin G; kDa: Kilodaltons; LRP/LR: Laminin receptor precursor/ laminin receptor; MTT: 3-(4,5-dimethylthiazol-2-yl)-2,5-diphenyltetrazolium bromide; PAGE: Polyacrylamide gel electrophoresis; PBS: Phosphate buffered saline; PCA: Protocatechuic acid; PI: Propidium iodide; PI3K: Phosphoinositide 3kinase; PS: Phosphatidyl serine; PVDF: Polyvinylidene fluoride; RLUC: Renilla luciferase; RNA: Ribonucleic acid; Rpm: Revolutions per minute; RPSA: Ribosomal protein SA; SDS: Sodium dodecyl sulfate; siRNA: Small interfering RNA; TEMED: Tetramethylethylenediamine

\section{Acknowledgements}

We thank Affimed Therapeutics GmbH, Heidelberg, Germany for providing antibody lgG1-iS18. We thank Carryn J. Chetty for guidance and knowledge on the topic.

\section{Funding}

This work is based upon research supported by the National Research Foundation (NRF), the Republic of South Africa (RSA). Grant Numbers 99061, 92745 and 109298. Any opinions, findings and conclusions or recommendations expressed in this material are those of the author(s), and therefore, the National Research Foundation does not accept any liability in this regard thereto. Financial support was received from the South African Medical Research Council (SAMRC) under the Wits Common Epithelial Cancer Research Centre (CECRC) grant. Any opinions, findings and conclusions or recommendations expressed in this material are those of the author(s), and therefore, the SAMRC does not accept any liability in this regard thereto. Financial support was further received from the Cancer Association of South Africa (CANSA). Any opinions, findings and conclusions or recommendations expressed in this material are those of the author(s), and therefore, CANSA does not accept any liability in this regard thereto.

\section{Availability of data and materials}

All data generated or analysed during this study are included in this published article [and its Additional file 1].

\section{Authors' contributions}

SFTW conceptualised and designed the study. LV performed experiments. LV and TMR analysed the data. EF and SFTW edited the manuscript. All authors have read and approved this version of the manuscript, and confirm that this is the case.

Ethics approval and consent to participate Not applicable.

\section{Competing interests}

The authors declare that they have no competing interests.

\section{Publisher's Note}

Springer Nature remains neutral with regard to jurisdictional claims in published maps and institutional affiliations. 


\section{Received: 8 December 2017 Accepted: 18 May 2018}

\section{Published online: 29 May 2018}

\section{References}

1. Stewart B, Wild CP. World cancer report 2014. In: Health; 2017.

2. Graham A, Adeloye D, Grant L, Theodoratou E, Campbell H. Estimating the incidence of colorectal cancer in Sub-Saharan Africa: A systematic analysis. J Glob Health. 2012;2(2):020404

3. Hanahan D, Weinberg RA. The hallmarks of cancer. cell. 2000;100(1):57-70.

4. Vania L, Chetty CJ, Ferreira E, Weiss SF. Anti-LRP/LR-specific antibody igG1 iS18 significantly impedes adhesion and invasion in early-and late-stage colorectal carcinoma cells. Mol Med. 2016;22:664.

5. Rebelo TM, Chetty CJ, Ferreira E, Weiss SF. Anti-LRP/LR-specific antibody IgG1-iS18 impedes adhesion and invasion of pancreatic cancer and neuroblastoma cells. BMC Cancer. 2016;16(1):917.

6. Munien C, Rebelo TM, Ferreira E, Weiss SF. IgG1-iS18 impedes the adhesive and invasive potential of early and late stage malignant melanoma cells. Exp Cell Res. 2017:351(2):135-41.

7. Omar A, Reusch U, Knackmuss S, Little M, Weiss SF. Anti-LRP/LR-specific antibody lgG1-iS18 significantly reduces adhesion and invasion of metastatic lung, cervix, colon and prostate cancer cells. J Mol Biol. 2012:419(1):102-9.

8. Chetty C, Khumalo T, Dias BDC, Reusch U, Knackmuss S, Little M, Weiss SF. Anti-LRP/LR specific antibody lgG1-iS18 impedes adhesion and invasion of liver cancer cells. PLoS One. 2014;9(5):e96268.

9. Khumalo T, Reusch U, Knackmuss S, Little M, Veale RB, Weiss SF. Adhesion and invasion of breast and oesophageal cancer cells are impeded by antiLRP/LR-specific antibody lgG1-iS18. PLoS One. 2013;8(6):e66297.

10. Digiacomo V, Gando IA, Venticinque L, Hurtado A, Meruelo D. The transition of the 37-kDa laminin receptor (RPSA) to higher molecular weight species: SUMOylation or artifact? Cell Mol Biol Lett. 2015;20(4):571-85.

11. Weiss SFT. Bad boy with a twist: targeting the $37 \mathrm{kDa} / 67 \mathrm{kDa}$ laminin receptor for treatment of Cancer and neurodegenerative diseases and for changing telomere dynamics. Cell \& Cellular Life Sciences Journal. 2017:2(2):1-3.

12. Nelson J, McFerran NV, Pivato G, Chambers E, Doherty C, Steele D, Timson DJ. The $67 \mathrm{kDa}$ laminin receptor: structure, function and role in disease. Biosci Rep. 2008;28(1):33-48.

13. Aznavoorian S, Stracke ML, Krutzsch H, Schiffmann E, Liotta LA. Signal transduction for chemotaxis and haptotaxis by matrix molecules in tumor cells. J Cell Biol. 1990;110(4):1427-38.

14. Khusal R, Dias BDC, Moodley K, Penny C, Reusch U, Knackmuss S, Little M, Weiss SF. In vitro inhibition of angiogenesis by antibodies directed against the $37 \mathrm{kDa} / 67 \mathrm{kDa}$ laminin receptor. PLoS One. 2013;8(3):e58888.

15. Givant-Horwitz V, Davidson B, Reich R. Laminin-induced signaling in tumor cells. Cancer Res. 2004:64(10):3572-9.

16. O'Donohue M-F, Choesmel V, Faubladier M, Fichant G, Gleizes P-E. Functional dichotomy of ribosomal proteins during the synthesis of mammalian 40S ribosomal subunits. J Cell Biol. 2010;190(5):853-66.

17. Venticinque L, Meruelo D. Comprehensive proteomic analysis of nonintegrin laminin receptor interacting proteins. J Proteome Res. 2012;11(10):4863-72

18. Scheiman J, Tseng JC, Zheng Y, Meruelo D. Multiple functions of the 37/67kd laminin receptor make it a suitable target for novel cancer gene therapy. Mol Ther. 2010;18(1):63-74

19. Wang K-S, Kuhn RJ, Strauss EG, Ou S, Strauss JH. High-affinity laminin receptor is a receptor for Sindbis virus in mammalian cells. J Virol. 1992; 66(8):4992-5001.

20. Jovanovic K, Gonsalves D, Da Costa Dias B, Moodley K, Reusch U, Knackmuss S, Penny C, Weinberg MS, Little M, Weiss SF. Anti-LRP/LR specific antibodies and shRNAs impede amyloid beta shedding in Alzheimer's disease. Sci Rep. 2013;3:2699.

21. Da Costa Dias B, Jovanovic K, Gonsalves D, Moodley K, Reusch U, Knackmuss S, Weinberg MS, Little M, Weiss SF. The 37kDa/67kDa laminin receptor acts as a receptor for Abeta42 internalization. Sci Rep. 2014;4:5556.

22. Pinnock EC, Jovanovic K, Pinto MG, Ferreira E, Dias BDC, Penny C, Knackmuss S, Reusch U, Little M, Schatzl HM. LRP/LR antibody mediated rescuing of amyloid- $\beta$-induced cytotoxicity is dependent on $\operatorname{PrPc}$ in Alzheimer's disease. J Alzheimers Dis. 2016;49(3):645-57.
23. Leucht C, Simoneau S, Rey C, Vana K, Rieger R, Lasmézas Cl, Weiss S. The 37 $\mathrm{kDa} / 67 \mathrm{kDa}$ laminin receptor is required for PrP Sc propagation in scrapieinfected neuronal cells. EMBO Rep. 2003;4(3):290-5.

24. Naidoo K, Malindisa ST, Otgaar TC, Bernert M, Dias BDC, Ferreira E, Reusch U, Knackmuss S, Little M, Weiss SF. Knock-down of the $37 \mathrm{kDa} / 67 \mathrm{kDa}$ laminin receptor LRP/LR impedes telomerase activity. PLoS One. 2015;10(11): e0141618.

25. Otgaar TC, Ferreira E, Malindisa S, Bernert M, Letsolo BT, Weiss SF. 37 kDa LRP:: FLAG enhances telomerase activity and reduces senescent markers in vitro. Oncotarget. 2017;8(49):86646.

26. Moodley K, Weiss SF. Downregulation of the non-integrin laminin receptor reduces cellular viability by inducing apoptosis in lung and cervical cancer cells. PLoS One. 2013:8(3):e57409.

27. Susantad T, Smith DR. siRNA-mediated silencing of the $37 / 67-\mathrm{kDa}$ high affinity laminin receptor in Hep3B cells induces apoptosis. Cellular \& molecular biology letters. 2008;13(3):452.

28. Khumalo T, Ferreira E, Jovanovic K, Veale RB, Weiss SF. Knockdown of LRP/LR induces apoptosis in breast and oesophageal cancer cells. PLoS One. 2015;10(10):e0139584.

29. Chetty CJ, Ferreira E, Jovanovic K, Weiss SF. Knockdown of LRP/LR induces apoptosis in pancreatic cancer and neuroblastoma cells through activation of caspases. Exp Cell Res. 2017:360(2):264-72.

30. Rebelo TM, Vania L, Ferreira E, Weiss SF. siRNA-mediated LRP/LR knockdown reduces cellular viability of malignant melanoma cells through the activation of apoptotic caspases. Exp Cell Res. 2018;368(1):1-12.

31. Wong RS. Apoptosis in cancer: from pathogenesis to treatment. J Exp Clin Cancer Res. 2011;30(1):87.

32. Wang B, Yuan H, Zhu C, Yang Q, Lv F, Liu L, Wang S. Polymer-drug conjugates for intracellar molecule-targeted photoinduced inactivation of protein and growth inhibition of cancer cells. Sci Rep. 2012;2:766.

33. Lee MJ, Song HJ, Jeong JY, Park SY, Sohn UD. Anti-oxidative and antiinflammatory effects of QGC in cultured feline esophageal epithelial cells. The Korean Journal of Physiology \& Pharmacology. 2013;17(1):81-7.

34. Hafiz S, Dennis JC, Schwartz D, Judd R, Tao YX, Khazal K, Akingbemi B, Mo $X-L$, Abdel-Mageed AB, Morrison E. Expression of melanocortin receptors in human prostate cancer cell lines: MC2R activation by ACTH increases prostate cancer cell proliferation. Int J Oncol. 2012:41(4):1373-80.

35. Kumar V, Tripathi V, Jahan S, Agrawal M, Pandey A, Khanna V, Pant A. Lead intoxication synergies of the ethanol-induced toxic responses in neuronal cells_PC12. Mol Neurobiol. 2015;52(3):1504-20.

36. Salama RH, Muramatsu H, Zou K, Inui T, Kimura T, Muramatsu T. Midkine binds to $37-k D a$ laminin binding protein precursor, leading to nuclear transport of the complex. Exp Cell Res. 2001;270(1):13-20.

37. Tsutsui J-i, Kadomatsu K, Matsubara S, Nakagawara A, Hamanoue M, Takao S, Shimazu H, Ohi Y, Muramatsu T. A new family of heparin-binding growth/differentiation factors: increased midkine expression in Wilms' tumor and other human carcinomas. Cancer Res. 1993:53(6):1281-5.

38. Kinoshita K, Kaneda Y, Sato M, Saeki Y, Wataya-Kaneda M, Hoffmann A, Kaneda Y. LBP-p40 binds DNA tightly through associations with histones H2A, H2B, and H4. Biochem Biophys Res Commun. 1998:253(2):277-82.

39. Shoieb AM, Elgayyar M, Dudrick PS, Bell JL, Tithof PK. In vitro inhibition of growth and induction of apoptosis in cancer cell lines by thymoquinone. Int J Oncol. 2003;22(1):107-13.

40. Shi Y. Mechanisms of caspase activation and inhibition during apoptosis Mol Cell. 2002;9(3):459-70.

41. Sun L, Liu L, Liu X, Wang Y, Li M, Yao L, Yang J, Ji G, Guo C, Pan Y. MGr1-ag/ 37LRP induces cell adhesion-mediated drug resistance through FAK/PI3K and MAPK pathway in gastric cancer. Cancer Sci. 2014;105(6):651-9.

42. Malygin AA, Babaylova ES, Loktev VB, Karpova GG. A region in the Cterminal domain of ribosomal protein SA required for binding of SA to the human 40 S ribosomal subunit. Biochimie. 2011;93(3):612-7.

43. Roy S, Nicholson DW. Cross-talk in cell death signaling. J Exp Med. 2000; 192(8):F21-6.

44. Huerta S, Goulet EJ, Livingston EH. Colon cancer and apoptosis. Am J Surg. 2006;191(4):517-26.

45. Hotchkiss RS, Nicholson DW. Apoptosis and caspases regulate death and inflammation in sepsis. Nat Rev Immunol. 2006;6(11):813.

46. Chen $\mathrm{Q}$, Gong B, Almasan A. Distinct stages of cytochrome c release from mitochondria: evidence for a feedback amplification loop linking caspase activation to mitochondrial dysfunction in genotoxic stress induced apoptosis. Cell Death Differ. 2000;7(2):227. 